Burnell, K., George, M. J., Vollet, J. W., Ehrenreich, S. E., \& Underwood, M. K. (2019). Passive social networking site use and well-being: The mediating roles of social comparison and the fear of missing out. Cyberpsychology: Journal of Psychosocial Research on Cyberspace, 13(3), article 5. http://dx.doi.org/10.5817/CP2019-3-5

\title{
Passive Social Networking Site Use and Well-Being: The Mediating Roles of Social Comparison and the Fear of Missing Out
}

\author{
Kaitlyn Burnell1 ${ }^{1}$, Madeleine J. George ${ }^{2}$, Justin W. Vollet ${ }^{1}$, Samuel E. Ehrenreich ${ }^{3}$, \& Marion K. Underwood ${ }^{2}$ \\ ${ }^{1}$ School of Behavioral and Brain Sciences, The University of Texas at Dallas, Richardson, TX, United States \\ 2 Department of Psychological Sciences, Purdue University, West Lafayette, IN, United States \\ ${ }^{3}$ College of Education, University of Nevada, Reno, NV, United States
}

\begin{abstract}
Passively browsing social networking sites (SNSs) correlates with poorer well-being (Verduyn, Ybarra, Résibois, Jonides, \& Kross, 2017). However, less research has been conducted that fully examines what factors may mediate this association. In particular, both online social comparison and the fear of missing out (FoMO) may play roles in how passive SNS browsing relates to depressive symptoms and self-perceptions. The current study adds to the literature by investigating how passive use relates to these outcomes through social comparison and FoMO. For an ethnically diverse sample of college students $\left(N=717, M_{\text {age }}=21.47, S D_{\text {age }}=4.64,69 \%\right.$ female), passively using SNSs positively predicted social comparison, which was positively related to FoMO, which in turn positively predicted depressive symptoms, and negatively predicted global self-worth, self-perceived physical appearance, and self-perceived social acceptance. These findings suggest that social comparison and FoMO play a role in the link between passive SNS use, depressive symptoms, and self-perceptions, and that FoMO could result from online social comparison.
\end{abstract}

Keywords: Social networking sites; passive use; online social comparison; fear of missing out; well-being

\section{Introduction}

Social networking sites (SNSs) are widely used by U.S. adults, with $69 \%$ using Facebook, 37\% using Instagram, 24\% using Snapchat, and 22\% using Twitter (Perrin \& Anderson, 2019). SNSs can have differential effects upon wellbeing depending on how they are used. For instance, active SNS use (i.e., uploading content) is suspected to be associated with improved subjective well-being, whereas passive SNS browsing (i.e., browsing without direct social interaction) is suspected to be particularly harmful for subjective well-being (Verduyn, Ybarra, Résibois, Jonides, \& Kross, 2017). Moreover, studies suggest that young people generally spend most of their time on SNS platforms passively browsing (Pempek, Yermolayeva, \& Calvert, 2009), with one study finding that participants typically passively browsed Facebook 50\% more than they use it actively (Verduyn et al., 2015). This further bolsters the need to understand associations with this activity.

People tend to engage in highly positive self-presentation online (Vogel \& Rose, 2016), which may be problematic for passive browsers in two primary ways. First, others' posts on SNSs may depict positive social events (Hu, Manikonda, \& Kambhampati, 2014; Underwood \& Ehrenreich, 2017). Thus, browsing this content may exacerbate the tendency to perceive that others have better social lives than oneself (Whillans, Christie, Cheung, Jordan, \& Chen, 2017) and reduce feelings of belonging (Whillans \& Chen, 2018). Second, the physical appearance of individuals depicted on SNSs may be enhanced, which could have implications for passive browsers' selfperceptions, namely in terms of self-perceived physical attractiveness (Fardouly \& Vartanian, 2016). Exposure to these types of content may be harmful through two mechanisms: a) by evoking harmful social comparisons, and 
b) by activating feelings of missing out. The goal of the current research was to explore links between passive SNS use, social comparison, and the fear of missing out (FoMO), with depressive symptoms and different domains of self-perceptions. We examined one broad index of self-perceptions (i.e., global self-worth) and two domains of self-perceptions that are particularly relevant to SNSs (i.e., self-perceived social acceptance and physical appearance).

\section{Social Comparison}

Social comparison theory suggests that in the absence of objective information, people have an innate drive to compare the self to others, often in an attempt to obtain an accurate self-evaluation (Festinger, 1954). SNS platforms provide a wealth of easily accessible information about others, and can therefore serve as a new avenue for people to engage in social comparison processes. As aforementioned, SNS content tends to be highly curated and focused on promoting a positive self-image (Vogel \& Rose, 2016), and therefore might elicit social comparisons that are predominantly upward in nature. Upward social comparisons, or the comparison of one's self to those perceived to be better off, may impair individuals' subjective well-being if these comparisons elicit negative feelings such as feelings of depression, envy, or resentment (Smith, 2000).

Few studies have directly tested the pathway between passive SNS browsing, upward social comparison, and lower well-being. One study investigated this path by examining feelings of envy, a prominent upward social comparison emotion (Smith, 2000), and found that passive Facebook use was indirectly related to depressive symptoms through envy (Tandoc, Ferrucci, \& Duffy, 2015). In addition, numerous studies have revealed links between specific components of this pathway. Passively browsing Facebook is associated with a greater tendency to engage in social comparisons on Facebook (Rousseau, Eggermont, \& Frison, 2017). In turn, making upward social comparisons on Facebook is linked with lower life satisfaction (de Vries \& Kühne, 2015; Gerson, Plagnol, \& Corr, 2016) and self-esteem (Lee, 2014; Vogel, Rose, Roberts, \& Eckles, 2014), as well as greater anxiety symptoms (Lee, 2014) and depressive symptoms (Chow \& Wan, 2017; Lee, 2014). Importantly, a study with an experience sampling design found that passively browsing Facebook was associated with lower affective well-being, which was mediated by feelings of envy (Verduyn et al., 2015). Thus, this study provides direct support for a pathway between passive SNS browsing, increased upward social comparison, and reduced well-being. Moreover, the tendency to engage in social comparisons on SNSs, regardless of the direction of the comparison (e.g., upward), is associated with poorer well-being. Specifically, engaging in social comparison on SNSs in general (Hanna et al., 2017) and on Facebook (Lee, 2014) is linked with poorer self-esteem and greater depressive and anxiety symptoms. Social comparison on Instagram is linked with poorer self-esteem (Stapleton, Luiz, \& Chatwin, 2017).

Experimental work has also provided evidence for links in this pathway. Browsing Facebook has been found to increase negative mood (Fardouly, Diedrichs, Vartanian, \& Halliwell, 2015), decrease affective well-being at the end of the day (although not immediately after browsing; Verduyn et al., 2015), and, for those high in their tendency to engage in social comparisons, reduce self-perceptions and self-esteem, and increase negative affect balance (Vogel, Rose, Okdie, Eckles, \& Franz, 2015).

SNS browsing and social comparison may differentially influence various domains of self-perceptions, which has received considerably less attention. This gap is notable, as self-perceptions are important for understanding effects of social comparison, and social comparisons can guide self-evaluations (Festinger, 1954). In particular, SNS browsing and SNS social comparisons may be relevant to self-perceived social acceptance and physical appearance. Social acceptance is relevant as SNS users may upload content depicting social events (Hu et al., 2014; Underwood \& Ehrenreich, 2017), including events that may exclude others (Lenhart, 2015), potentially hurting selfperceived social acceptance for browsers who were excluded from these events. Physical appearance is relevant as ample research has demonstrated links between SNS use and body image concerns as a result of the attractive images others post online (for a review, see Fardouly \& Vartanian, 2016). Moreover, both self-perceived social acceptance and physical appearance may be hurt by quantifiable indices of approval that are rampant on SNSs. For example, a filtered, appearance-enhancing post depicting a positive social event that receives many "likes" may be especially likely to evoke social comparisons and consequently hurt self-perceived social acceptance and/or physical appearance (e.g., Nesi, Choukas-Bradley, \& Prinstein, 2018). To our knowledge, only one study has examined the links between these self-perceptions and SNS social comparisons, which found that Facebook 
upward social comparisons is associated with poorer self-perceived physical appearance and social acceptance (de Vries \& Kühne, 2015).

In addition to these specific domains of self-perceptions, we examined global self-worth, which assesses one's perceptions of the self as a whole. Making SNS social comparisons may be relevant to global self-worth as upward social comparisons could lead to the perception that others are better off than the self (e.g., Chou \& Edge, 2012), and therefore trigger negative self-evaluations. Finally, we included depressive symptoms. As discussed above, depressive symptoms is a well-established correlate with SNS social comparison, and so we included it to examine the role these symptoms may have in our full model.

\section{Fear of Missing Out}

The fear of missing out (FoMO) may also play an important role in the associations between passive SNS use, social comparison, and depressive symptoms and self-perceptions. FoMO is the "pervasive apprehension that others might be having rewarding experiences from which one is absent" (Przybylski, Murayama, DeHaan, \& Gladwell, 2013 , p. 1841). Given that FoMO requires perceiving that others are having more rewarding experiences than the self, and therefore that others are better off than the self, it can be considered a type of upward social comparison. Indeed, FoMO is strongly linked to one's general tendency to engage in social comparison (Reer, Tang, \& Quandt, 2019).

FoMO is intrinsically linked to SNS use, with Przybylski and colleagues (2013) suggesting that FoMO is characterized by low need satisfaction that compels people to continuously check SNSs to feel connected to others. FoMO has consistently been found to be related to greater levels of SNS use (Baker, Krieger, \& LeRoy, 2016; Blackwell, Leaman, Tramposch, Osborne, \& Liss, 2017; Przybylski et al., 2013). Although it is yet to be tested how FoMO relates to specifically passive SNS use, there is reason to suspect that FoMO may be particularly related to this type of SNS use, as passive use can fulfill the need to monitor peers' activities.

There is also reason to suspect that FoMO is linked to greater depressive symptoms and poorer self-perceptions. A key conceptual component of FoMO is a high degree of negative affect, as FoMO requires some anxiety by definition (Przybylski et al., 2013). Cross-sectionally, FoMO has small-to-moderate correlations with numerous facets of poorer well-being, including lower life satisfaction and mood (Przybylski et al., 2013), depressive symptoms (Baker et al., 2016; Elhai, Levine, Dvorak, \& Hall, 2016; Elhai et al., 2018), anxiety symptoms (Elhai et al., 2016; Elhai et al., 2018), and stress, rumination, and proneness to boredom (Elhai et al., 2018). Importantly, the size of these correlations suggests that although negative affect is an important characteristic of FoMO, the association is not so strong as to suggest that they are indistinct. Although little research has examined how FoMO may fit in a pathway between SNS use and well-being, one study tested the notion that SNS use may trigger feelings of FoMO, which in turn may reduce well-being. This longitudinal study provided evidence that greater SNS use was related to increased FoMO six months later, which in turn was related to decreased self-esteem (Buglass, Binder, Betts, \& Underwood, 2017). Given that much previous research has focused on how existing feelings of FoMO may drive SNS use (Przybylski et al., 2013), this study is important as it provides support for the notion that SNS use may trigger feelings of FoMO which in turn may have negative repercussions for subjective well-being.

As noted in the literature reviewed above, the link between FoMO and depressive symptoms is well established. However, investigations have yet to expand investigations of FoMO to self-perceptions. Because SNSs may be used to depict positive social events (Hu et al., 2014; Underwood \& Ehrenreich, 2017), FoMO may have a particularly strong connection with self-perceived social acceptance. The perception that others are having more rewarding experiences than the self may be especially intertwined with others' social experiences, and thus hurt selfperceived social acceptance if these social experiences are perceived to be superior than one's own. Likewise, those high in FoMO may report lower levels of global self-worth, as they may believe that they, as a person, pale in comparison to those who seemingly have more rewarding experiences. Links between FoMO and self-perceived physical appearance are not immediately clear, and therefore these associations will be examined as exploratory. 


\section{Considering Different SNS Platforms}

The vast majority of studies reviewed above focus on Facebook. Although highly informative, these studies should be expanded for two reasons. First, researchers have previously suggested that certain social processes may occur differently depending on which SNS platform is being used (Reich, 2017), and therefore generalizing findings from one SNS to all SNSs may be inappropriate (Valenzuela, Park, \& Kee, 2009; Wilson, Gosling, \& Graham, 2012). Thus, social processes such as social comparison and FoMO, and associations that these constructs have with other variables may be different depending on which SNS platform is being examined. For example, social comparison may be particularly relevant to image-centric platforms such as Instagram, especially in the context of body image concerns (e.g., Marengo, Longobardi, Fabris, \& Settanni, 2018). Moreover, given that uploading pictures of friends is common on Instagram (Hu et al., 2014), social comparison may also be particularly potent in the context of social acceptance. Similarly, Facebook may also be used to detail social occasions, particularly in terms of tagging others in content and using the platform to plan social events. In contrast, Snapchat is often used to convey humorous content (Piwek \& Joinson, 2016), and Twitter has a micro-blog format, and therefore social comparison and FoMO processes on these two platforms may be qualitatively different from Facebook and Instagram. Second, nationally representative data suggest that Facebook is no longer the most dominant SNS platform among younger people, who tend to show preference for other sites, such as Instagram and Snapchat (Anderson \& Jiang, 2018; Perrin \& Anderson, 2019). Taken together, SNS investigations should be extended to examine multiple platforms, in order to a) distinguish whether associations may differ across platforms, and b) to keep up with constantly evolving trends in SNS use. To address this, we examined differences in our hypothesized path across four major SNS platforms (Facebook, Instagram, Twitter, and Snapchat) as an exploratory aim in our investigation.

\section{The Present Study}

This study investigated how passive SNS use relates to social comparison, FoMO, and depressive symptoms and self-perceptions. This study expands existing research in several ways. First, this is one of the few studies that tests a complete path between passive SNS use, social comparison processes, and depressive symptoms and selfperceptions. Although this pathway has been suggested by other researchers (Verduyn et al., 2017), few studies have explicitly tested it (see Tandoc et al., 2015, Verduyn et al., 2015, for exceptions). Second, to the best of our knowledge, this is the first study that considers the role of FoMO in this path. Considering our speculation of how FoMO could be a particular type of social comparison, it is important to understand how FoMO may fit in this path. Third, passive SNS use was examined across four platforms. Most previous research has focused on Facebook, despite platforms such as Snapchat and Instagram growing in popularity among young adults (Perrin \& Anderson, 2019). Fourth, we consider different types of self-perceptions, namely self-perceived physical appearance and selfperceived social acceptance. These self-perceptions, as noted above, may have special relevance for SNSs.

There are various possibilities for the sequential ordering of passive SNS use, social comparison, FoMO, and depressive symptoms and self-perceptions. Our primary hypothesis revolves around the notion that SNSs may fuel feelings of FoMO, as passively browsing the positive content that others post online may then trigger the fear that others are having more rewarding experiences than the self (Buglass et al., 2017). Thus, FoMO would follow passive SNS use. Moreover, if FoMO can be considered a type of upward social comparison, FoMO would follow social comparison. In particular, it is expected that after one begins the process of passive browsing, he or she must first engage in comparative processes, in general, before making explicit upward-type comparisons. Detrimental effects upon depressive symptoms and self-perceptions would then occur after these upward comparisons are made.

Alternatively, people with higher levels of FoMO may turn to SNSs to fulfill their need for connection (Przybylski et al., 2013). Thus, FoMO would precede passive SNS use, which in turn may lead to greater social comparison, and subsequently increased depressive symptoms and lower self-perceptions. The current research therefore tests two opposing possibilities:

H1A: Passively using SNSs positively predicts social comparison, which in turn predicts higher levels of FoMO, which subsequently predicts greater depressive symptoms and poorer self-perceptions. 
H1B: FoMO positively predicts passively using SNSs, which in turn predicts increased social comparison, which subsequently predicts greater depressive symptoms and poorer self-perceptions.

In addition, researchers have previously suggested that poorer well-being may trigger people to use SNSs as a method of mood management (Frison \& Eggermont, 2017). Thus, the current research tests this pathway as an exploratory analysis.

H1C: Greater depressive symptoms and poorer self-perceptions predicts greater passive SNS use, which in turn predicts increased social comparison, which subsequently positively predicts FoMO.

\section{Method}

\section{Participants}

A convenience sample of 744 undergraduate students was recruited from a southwestern public university during the $2016-2017$ academic year $\left(M_{\text {age }}=21.47, S D_{\text {age }}=4.64\right.$, Range age $=18-59 ; 69 \%$ female; 39\% Asian, 36\% White, $16 \%$ Hispanic/Latinx, 6\% Black, 2\% Other). A final sample of 717 students completed all key survey items. Participants were recruited via online advertisements for class research credit and completed the surveys online via Qualtrics. Participants were provided a battery of assessments that examined digital technology use and behaviors. Only measures relevant to the current research are reported. All study procedures were approved by The University of Texas at Dallas Institutional Review Board (IRB \#17-15).

\section{Measures}

Passive social networking site use. For Facebook, Instagram, Twitter, and Snapchat, participants answered an open-ended question that asked: "How many minutes per day do you spend viewing others' profiles?". Participants were also given the option to write-in other platforms they passively browse, and how often they browse each one; however, these write-ins were uncommon. On average, students spent 14 minutes per day browsing Facebook, 17 minutes on Instagram, 12 minutes on Twitter, and 19 minutes on Snapchat. Passive SNS use was summed across platforms, averaging about one hour. Given that most time on SNSs is spent browsing (Pempek et al., 2009; Verduyn et al., 2015), this estimate is similar to previous research suggesting that young adults typically spend one hour per day using SNSs as a whole (Shensa, Sidani, Lin, Bowman, \& Primack, 2016).

Social comparison. The ten-item Comparison and Feedback-Seeking scale (Nesi, 2014; Nesi \& Prinstein, 2015) was used to measure participants' engagement of technology-based social comparisons and feedback-seeking behaviors. We chose this scale as we were interested in evaluating general comparison tendencies as they occur on digital platforms. Participants answered items using a 5-point Likert-type scale (1: "Not at all true", 5: "Extremely true"). The original scale was developed for adolescents, and captures both social comparison and feedbackseeking behaviors, as these constructs are key self-evaluative processes for adolescents (Nesi, 2014). Although the original study considered social comparison and feedback-seeking together, a confirmatory factor model in our sample suggested two distinct social comparison and feedback-seeking factors, $\chi^{2}(26)=212.300, p<.001, C F I=$ $0.932, R M S E A=.099,90 \% \mathrm{Cl}[.088, .113], \mathrm{SRMR}=.049^{1}$. As theory suggests that social comparison may be particularly relevant for understanding links between passive SNS use, depressive symptoms, and self-worth, and because FoMO can be reasoned to be a type of social comparison, we opted to focus on the four social comparison items of the measure. A confirmatory factor analysis for the social comparison items demonstrated acceptable fit, $\chi^{2}(2)=11.283, p=.004, C F I=0.989, R M S E A=.080,90 \% \mathrm{Cl}[.039, .129], \mathrm{SRMR}=.017$. A sample item is "I use electronic interaction to compare my life with other people's lives" $(a=.86)$.

Fear of missing out. FoMO was measured using Przybylski et al.'s (2013) ten-item Fear of Missing Out scale (e.g., "I fear others have more rewarding experiences than $\mathrm{me}^{\prime} ; \mathrm{a}=.90$ ). Participants answered items using a 5-point Likert-type scale (1: "Not at all true of me", 5: "Extremely true of me"). This scale has shown content validity (Przybylski et al., 2013) and is currently the main assessment of FoMO in the field.

Depressive symptoms. Depressive symptoms were measured with the nine-item Patient Health Questionnaire (PHQ-9; Kroenke, Spitzer, \& Williams, 2001). Participants indicated how often, in the last two weeks, they had been 
bothered by a list of problems (e.g., "Feeling down, depressed, or hopeless"; a = .91), utilizing a 4-point Likert-type scale (1: "Not at all", 4: "Nearly every day"). The PHQ-9 is high in criterion, construct, and external validity, as well as test-retest reliability (Kroenke et al., 2001).

Self-perceptions. Participants completed three subscales of the Self-Perception Profile for College Students (Neemann \& Harter, 2012) assessing Global Self-Worth (e.g., "Some students like the kind of person they are BUT Other students wish that they were different"; $a=.89$; six items), Social Acceptance (e.g., "Some students are not satisfied with their social skills BUT Other students think their social skills are just fine"; $a=.84$; four items), and Physical Appearance (e.g., "Some students wish their body was different BUT Other students like their body the way it is"; $a=.85$; four items). Participants were presented two statements, one exemplifying poorer self-perceptions and the other assessing positive self-perceptions. Participants picked which statement they were most like, and indicated if the statement was "Really true for me" or "Sort of true for me". Statements were combined into one 4point item, with lower values indicating poorer self-perceptions. The Self-Perception Profile for College Students is content valid and has demonstrated convergent validity (Neemann \& Harter, 2012).

Due to the inherent negative affect component to FoMO, a confirmatory factor analysis was run to distinguish FoMO from depressive symptoms and self-perceptions. It should be noted that because each measure has been previously tested and validated, the aim was not to validate the measurement model but to ensure that items load onto their assigned factor with little cross-loading onto other factors/constructs. A five factor confirmatory factor model with separate FoMO, depressive symptoms, social acceptance, self-worth, and physical appearance factors showed adequate fit, $\chi^{2}(515)=1424.451, p<.001, C F I=0.918, R M S E A=.050,90 \% \mathrm{Cl}[.047, .053]$, SRMR $=.054$.

\section{Analytical Approach}

A set of path models was specified in Mplus (version 8; Muthén \& Muthén, 2017) to investigate a pathway between composites of passive SNS use, social comparison, FoMO, and depressive symptoms and self-perceptions. Students were included in the models if they had completed the SNS estimates, social comparison items, FoMO items, and at least one outcome scale of depressive symptoms or self-perceptions $(N=717)$. Models were specified using maximum likelihood estimation and bootstrapping methods.

\section{Results}

\section{Hypothesized Models}

Descriptive statistics are reported in Table 1. To examine hypothesis $1 \mathrm{~A}$, a mediation path model was tested with passive SNS use predicting social comparison, social comparison predicting FoMO, and FoMO predicting depressive symptoms and self-perceptions (Figure 1). Model fit was good, $\chi^{2}(8)=27.570, p=.001, C F I=0.984$, RMSEA $=.058,90 \% \mathrm{Cl}[.036, .083], \mathrm{SRMR}=.031$. Social comparison and FoMO fully mediated the associations between passive SNS use and depressive symptoms (indirect effect via social comparison and FoMO, $\beta=.04, p<$ .001 ), global self-worth (indirect effect, $\beta=-.04, p<.001$ ), and social acceptance (indirect effect, $\beta=-.03, p<.001$ ). As mediators, both social comparison and FoMO were directly associated with students' self-perceived physical appearance $(\beta=-.18, p<.001$ and $\beta=-.16, p<.001$, respectively); however, the effect of social comparison on students' perception of their physical appearance was partially mediated by FoMO (indirect effect via social comparison and FoMo $\beta=-.02, p=.005$ ). Thus, hypothesis $1 \mathrm{~A}$ was supported.

To test hypothesis 1B, a mediation path model was tested with FoMO predicting passive SNS use, passive SNS use predicting social comparison, and social comparison predicting depressive symptoms and self-perceptions. Model fit was poor, $\chi^{2}(9)=382.110, p<.001, C F I=0.697, R M S E A=.240,90 \% \mathrm{Cl}[.220, .261]$, SRMR $=.143$. Thus, hypothesis $1 \mathrm{~B}$ was rejected. To examine hypothesis $1 \mathrm{C}$, a mediation path model was tested with depressive symptoms and self-perceptions predicting passive SNS use, which in turn predicted social comparison, which subsequently predicted FoMO. This model yielded poor fit, $\chi^{2}(9)=170.902, p<.001, C F I=0.687, R M S E A=.160,90 \% \mathrm{Cl}[.139, .181]$, $\mathrm{SRMR}=.141$, and therefore hypothesis $1 \mathrm{C}$ was rejected. 
Table 1. Descriptive Statistics and Correlations.

\begin{tabular}{|c|c|c|c|c|c|c|c|c|}
\hline & Mean (SD) & 1 & 2 & 3 & 4 & 5 & 6 & 7 \\
\hline 1. SNS Passive Browsing (minutes) & $57.93(93.66)$ & - & & & & & & \\
\hline 2. Social Comparison (1-5) & $2.68(1.07)$ & $.17^{* *}$ & - & & & & & \\
\hline 3. Fear of Missing Out (1-5) & $2.41(0.91)$ & $.17^{* *}$ & $.61^{* *}$ & - & & & & \\
\hline 4. Depressive Symptoms (1-4) & $1.86(0.69)$ & $.11^{*}$ & $.29^{* *}$ & $.34^{* *}$ & - & & & \\
\hline 5. Global Self-Worth (1-4) & $2.72(0.66)$ & -.02 & $-.31^{* *}$ & $-.36^{\star \star}$ & $-.52^{* \star}$ & - & & \\
\hline 6. Social Acceptance (1-4) & $2.54(0.68)$ & -.01 & $-.22^{\star *}$ & $-.26^{* *}$ & $-.29^{\star \star}$ & $.56^{\star \star}$ & - & \\
\hline 7. Physical Appearance (1-4) & $2.45(0.72)$ & -.04 & $-.32^{* *}$ & $-.27^{\star \star}$ & $-.38^{\star \star}$ & $.50^{\star \star}$ & $.34^{* *}$ & - \\
\hline
\end{tabular}

Note: $N=717 .{ }^{*} p<.01,{ }^{*} p<.001$

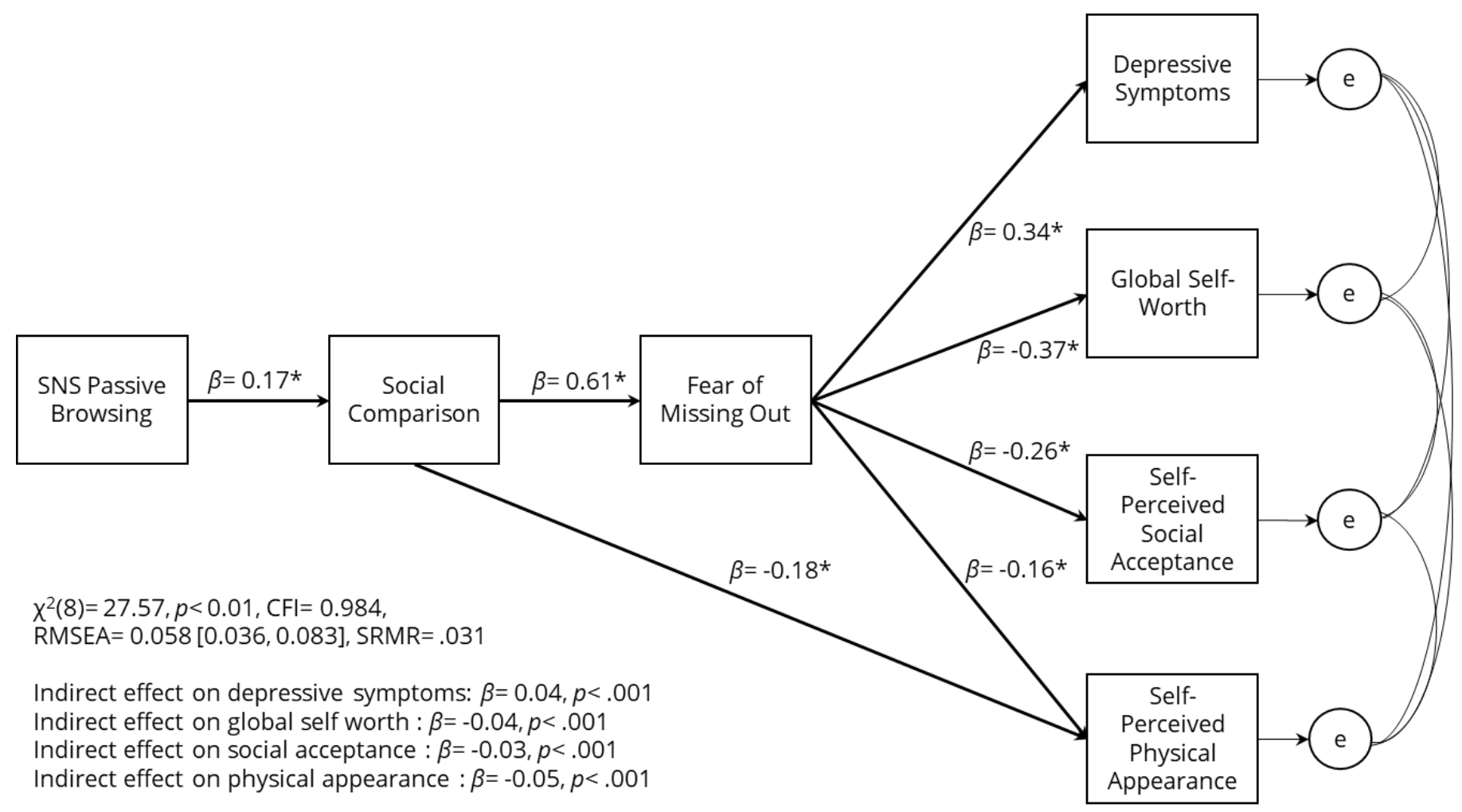

Figure 1. Mediation path model testing hypothesis 1A.

Note. ${ }^{*} p<.01$

\section{Exploratory Models}

Sensitivity analyses were conducted to test the robustness of the model depicted in hypothesis $1 \mathrm{~A}$. First, the model held when controlling for age, gender, and a measure of general SNS use (i.e., how often participants checked their SNS accounts daily), $\chi^{2}(8)=24.073, p=.002, C F I=0.988, R M S E A=.053,90 \% \mathrm{Cl}[.029, .078], \mathrm{SRMR}=.016$. Second, a series of multi-group equality constrained and unconstrained models by age, gender, and SNS checking were examined to check for model differences between groups. Model fit was acceptable when model paths were set to be equal by age (i.e., comparing 18-19 year olds versus 20+ year olds, to compare students earlier in their college education to those later), $\chi^{2}(23)=39.299, p=.018, C F I=0.987, R M S E A=.045,90 \% \mathrm{Cl}[.018, .068]$, SRMR $=$ .042 , by gender, $\chi^{2}(23)=54.347, p<.001, C F I=0.973, R M S E A=.065,90 \% \mathrm{Cl}[.043, .087], \mathrm{SRMR}=.056$, and for SNS users with high versus low checking, $\chi^{2}(23)=47.099, p=.002, C F I=0.980, R M S E A=.054,90 \% \mathrm{Cl}[.032, .076]$, SRMR $=.048$. In the unconstrained model, there was a weaker path from SNS passive browsing to social comparison for participants aged 18-19 $(\beta=.13, p=.05)$ and compared to the same path for participant aged $20+(\beta=.19, p=.01)$. When comparing men and women in the unconstrained model, the paths from social comparison and FoMO to perceptions of physical appearance were significant for women $(\beta=-.19, p<.001 ; \beta=-.18, p<.001)$ but not men $(\beta=-.10, p=.33 ; \beta=-.13, p=.21)$. Similarly, there was a significant path from FoMO to perceptions of physical appearance for participants with high SNS checking $(\beta=-.21, p=.001)$, but not for participants with low SNS 
checking ( $\beta=-.10, p=.10$ ). Together, these results suggests only minor differences in associations between the tested variables based on age, gender, and SNS checking.

Finally, separate analyses were conducted for passive SNS use on the four most popular individual platforms (Facebook, Instagram, Snapchat, and Twitter), rather than aggregating use. Each of these models demonstrated acceptable fit (Table 2 ). In terms of path coefficients, passively browsing Instagram was the strongest predictor of social comparison $(\beta=.17, p<.001)$, followed by Facebook $(\beta=.14, p=.001)$, Twitter $(\beta=.12, p<.001)$, and Snapchat $(\beta=.11, p=.002)$. All other path coefficients in the models did not differ greatly (Betas were all within .02 of each other and the model shown Figure 1).

Table 2. Fit Statistics for the Path Models Separated by SNS Platform.

\begin{tabular}{llllll}
\hline & $\mathbf{N}$ & Chi-Square & CFI & RMSEA & SRMR \\
\hline 1. Facebook & 689 & $\chi^{2}(8)=35.616 p<.001$ & 0.977 & $.071[.048, .095]$ & .035 \\
2. Instagram & 674 & $\chi^{2}(8)=24.722 p<.001$ & 0.985 & $.056[.032, .081]$ & .031 \\
3. Twitter & 675 & $\chi^{2}(8)=16.966 p=.031$ & 0.992 & $.041[.012, .068]$ & .027 \\
4. Snapchat & 651 & $\chi^{2}(8)=22.454 p=.004$ & 0.987 & $.053[.028, .079]$ & .031 \\
\hline
\end{tabular}

Note: All models used the same path model as shown in Figure 1.

\section{Discussion}

Results indicated that passively browsing SNSs positively predicts social comparison, which in turn is associated with higher levels of FoMO, which subsequently predicts lower levels of global self-worth, lower levels of selfperceived social acceptance and physical appearance, and higher levels of depressive symptoms. This study extends previous research (de Vries \& Kühne, 2015) by replicating links with self-perceptions in a large, ethnically diverse sample, by considering passive SNS use across platforms, and by including FoMO.

Social comparison theory (Festinger, 1954) suggests that people have an innate drive to compare the self to others in the absence of objective information. Researchers have previously suggested that social comparison, particularly upward social comparison, plays an important role on SNSs (Verduyn et al., 2017). In particular, passively browsing others' content on SNSs may elicit upward social comparisons, which may negatively impact well-being. Our findings provide support for this path. However, we also examined the role of FoMO, which can be considered a type of upward social comparison. The positioning of FoMO in the model suggests an important consideration for future work examining this construct. Researchers have suggested that FoMO motivates use of SNSs (Przybylski et al., 2013). The current research found that FoMO fit better following social comparison, rather than as a predictor of passive SNS use. FoMO may result in part from engaging in social comparison. Perhaps engaging in social comparison online fuels the perception that others are having more rewarding experiences, as one is attempting to gather social information in the context of the highly positive content others post online (Vogel \& Rose, 2016). This may further the belief that others have richer social lives than the self (e.g., Whillans et al., 2017), thus triggering feelings of FoMO. However, these conjectures are speculative due to the cross-sectional nature of the data, which does not permit drawing causal inferences regarding the ordering of the constructs in our model. Future longitudinal and experimental research is needed to determine temporality and causality.

Additionally, FoMO predicted greater levels of depressive symptoms, lower global self-worth, and lower selfperceived social acceptance and physical appearance, in line with previous research suggesting the FoMO is linked to poorer well-being (e.g., Przybylski et al., 2013). The reverse pathway examining depressive symptoms and selfperceptions predicting passive SNS use demonstrated poor fit, potentially suggesting that those lower in wellbeing may not be more likely to browse (e.g., Frison \& Eggermont, 2017); however, once again, longitudinal and experimental research is needed to more definitively determine the ordering of these constructs.

Regarding social acceptance, fearing that others are having more rewarding experiences may hurt an individual's own self-perceived social competence. As content on SNSs may detail social events from which an individual is excluded (Lenhart, 2015), those high in FoMO may be especially attuned to potential exclusion, and may feel less socially accepted. Additionally, both FoMO and social comparison directly predicted lower levels of self-perceived 
physical appearance. Given that SNS use can stoke appearance social comparisons (Fardouly \& Vartanian, 2016), individuals who are high on social comparison and thus frequently compare themselves to others may feel worse about their physical appearance after viewing highly positive SNS content. This relation may also be due to how several social comparison scale items were relevant to physical appearance (e.g., "I use electronic interaction to compare my body/shape with other people's bodies/shapes"). FoMO was also related to reduced self-perceived physical appearance. Perhaps FoMO may lead individuals to wonder why they are missing out, triggering a heightened sense of self-awareness. This may manifest as an appraisal of their physical appearance, due to the interpersonal benefits that might accompany being highly attractive. However, this is speculative and future research should examine this link further.

Our model was generally consistent across individuals, regardless of age, gender, and frequency of using SNSs. There were a few notable exceptions. First, the path between SNS browsing and social comparison was weaker for participants aged 18-19 compared to those who were at least 20-years-old. This is surprising, as social comparisons occur more frequently among younger individuals (Callan, Kim, \& Matthews, 2015). Future research should examine the mechanisms that may influence this path further. Second, the paths between social comparison and FoMO to self-perceived physical appearance were significant for women but not men. This is in line with previous research that suggests that social comparison is more strongly linked to body dissatisfaction for women compared to men (Myers \& Crowther, 2009). Last, FoMO was linked to increased self-perceived physical appearance for those who checked their SNS accounts more frequently, but not for those who checked their accounts less frequently. It could be that those who check SNSs more often may be more concerned and aware about their SNS presence. With this greater self-awareness, our speculated link between FoMO and self-perceived physical appearance may be strengthened: these individuals may wonder why they are missing out, leading to an appraisal of their physical appearance.

Our model was generally consistent across SNS platforms. Perhaps these platforms share key affordances, such as being able to see what others are doing and what types of feedback others receive, that might drive the relations with social comparison and FoMO. Interestingly, one difference did emerge among platforms, passive browsing was linked most strongly to social comparison on Instagram, followed by Facebook, Twitter, and then Snapchat. Researchers have previously suggested that image-centric platforms may be more conducive to eliciting social comparisons (namely, appearance-oriented comparisons; Marengo et al., 2018), and therefore this finding is unsurprising given that Instagram is entirely image-oriented. Facebook is also image-centric, although not to the same degree, and so it follows that the strength of the relation between passive browsing and social comparison would also be relatively strong. This is in contrast with Twitter, which encompasses more written content. One may expect that Snapchat would be more strongly related to social comparison than it was found to be given that it is also entirely image-oriented. However, Snapchat users report using the platform mainly to send humorous content (Piwek \& Joinson, 2016), and therefore the manifestations of social comparison Snapchat may be different compared to other platforms, especially Instagram, which mainly consists of highly curated content (Underwood \& Ehrenreich, 2017).

A number of limitations should be noted. First, this study is cross-sectional; longitudinal and experimental research are needed to determine the causal sequence of the examined constructs. Second, a convenience sample of college students was utilized, which may limit generalizability. Third, we did not counterbalance the presentation of the measures, and therefore results may have been influenced by survey ordering; however, the observed pathway was not in the same order as the measures were presented. Fourth, our assessment of passive SNS use consisted of single open-ended, self-reported items about viewing others' profiles on each platform. Future research should expand this assessment by asking about passive use in general (e.g., browsing newsfeeds) and by employing objective assessments of SNS use (e.g., device-recorded screen time). Fifth, social comparison and FoMO were statistically tested as state-like variables that follow engaging in passive SNS use. These variables could also be considered personality traits that drive an individual to use SNSs, although model fit when testing this with FoMO was poor. Future research should attempt to distinguish state and trait social comparison and FoMO, to understand this pathway further.

Ultimately, these findings suggest that social comparison and FoMO play important roles in understanding the link between passively browsing SNSs, depressive symptoms, and self-perceptions. The results also highlight how FoMO may be a type of upward social comparison. Being apprehensive that others are having more rewarding 
experiences may inherently require some degree of social comparison, as one is perceiving that others are, at least in the moment, better off than oneself. Additionally, these results provide new evidence regarding how FoMO relates to self-perceptions. Altogether, this study advances our understanding of how SNSs may be affecting users by highlighting how online social comparison and FoMO may adversely impact passive browsers.

\section{Notes}

1. The indices reported are after removing one poor feedback-seeking item ("I use electronic interaction to see what the "popular" kids think about me"). This item was likely poor in our sample as we recruited college students, for whom the meaning of popularity may differ from adolescents.

\section{References}

Anderson, M., \& Jiang, J. (2018, May 31). Teens, social media \& technology 2018. Pew Research Center. Retrieved from http://www.pewinternet.org/2018/05/31/teens-social-media-technology-2018/

Baker, Z. G., Krieger, H., \& LeRoy, A. S. (2016). Fear of missing out: Relationships with depression, mindfulness, and physical symptoms. Translational Issues in Psychological Science, 2, 275-282.

https://doi.org/10.1037/tps0000075

Blackwell, D., Leaman, C., Tramposch, R., Osborne, C., \& Liss, M. (2017). Extraversion, neuroticism, attachment style and fear of missing out as predictors of social media use and addiction. Personality and Individual Differences, 116, 69-72. https://doi.org/10.1016/j.paid.2017.04.039

Buglass, S. L., Binder, J. F., Betts, L. R., \& Underwood, J. D. M. (2017). Motivators of online vulnerability: The impact of social network site use and FOMO. Computers in Human Behavior, 66, 248-255.

https://doi.org/10.1016/j.chb.2016.09.055

Callan, M. J., Kim, H., \& Matthews, W. J. (2015). Age differences in social comparison tendency and personal relative deprivation. Personality and Individual Differences, 87, 196-199. https://doi.org/10.1016/j.paid.2015.08.003

Chou, H.-T. G., \& Edge, N. (2012). "They are happier and having better lives than I am": The impact of using Facebook on perceptions of others' lives. Cyberpsychology, Behavior, and Social Networking, 15, 117-121. https://doi.org/10.1089/cyber.2011.0324

Chow, T. S., \& Wan, H. Y. (2017). Is there any 'Facebook Depression'? Exploring the moderating roles of neuroticism, Facebook social comparison and envy. Personality and Individual Differences, 119, 277-282. https://doi.org/10.1016/j.paid.2017.07.032

de Vries, D. A., \& Kühne, R. (2015). Facebook and self-perception: Individual susceptibility to negative social comparison on Facebook. Personality and Individual Differences, 86, 217-221.

https://doi.org/10.1016/j.paid.2015.05.029

Elhai, J. D., Levine, J. C., Alghraibeh, A. M., Alafnan, A. A., Aldraiweesh, A. A., \& Hall, B. J. (2018). Fear of missing out: Testing relationships with negative affectivity, online social engagement, and problematic smartphone use. Computers in Human Behavior, 89, 289-298. https://doi.org/10.1016/j.chb.2018.08.020

Elhai, J. D., Levine, J. C., Dvorak, R. D., \& Hall, B. J. (2016). Fear of missing out, need for touch, anxiety and depression are related to problematic smartphone use. Computers in Human Behavior, 63, 509-516. https://doi.org/10.1016/j.chb.2016.05.079

Fardouly, J., Diedrichs, P. C., Vartanian, L. R., \& Halliwell, E. (2015). Social comparisons on social media: The impact of Facebook on young women's body image concerns and mood. Body Image, 13, 38-45.

https://doi.org/10.1016/j.bodyim.2014.12.002 
Fardouly, J., \& Vartanian, L. R. (2016). Social media and body image concerns: Current research and future directions. Current Opinion in Psychology, 9, 1-5. https://doi.org/10.1016/j.copsyc.2015.09.005

Festinger, L. (1954). A theory of social comparison processes. Human Relations, 7, 117-140. https://doi.org/10.1177/001872675400700202

Frison, E., \& Eggermont, S. (2017). Browsing, posting, and liking on Instagram: The reciprocal relationships between different types of Instagram use and adolescents' depressed mood. Cyberpsychology, Behavior, and Social Networking, 20,603-609. https://doi.org/10.1089/cyber.2017.0156

Gerson, J., Plagnol, A. C., \& Corr, P. J. (2016). Subjective well-being and social media use: Do personality traits moderate the impact of social comparison on Facebook? Computers in Human Behavior, 63, 813-822.

https://doi.org/10.1016/j.chb.2016.06.023

Hanna, E., Ward, L. M., Seabrook, R. C., Jerald, M., Reed, L., Giaccardi, S., \& Lippman, J. R. (2017). Contributions of social comparison and self-objectification in mediating associations between Facebook use and emergent adults' psychological well-being. Cyberpsychology, Behavior, and Social Networking, 20, 172-179.

https://doi.org/10.1089/cyber.2016.0247

Hu, Y., Manikonda, L., \& Kambhampati, S. (2014). What we Instagram: A first analysis of Instagram photo content and user types. In Proceedings of the Eighth International AAAI Conference on Weblogs and Social Media, 595-598.

Kroenke, K., Spitzer, R. L., \& Williams, J. B. W. (2001). The PHQ-9: Validity of a brief depression severity measure. Journal of General Internal Medicine, 16, 606-613. https://doi.org/10.1046/j.1525-1497.2001.016009606.x

Lee, S. Y. (2014). How do people compare themselves with others on social network sites?: The case of Facebook. Computers in Human Behavior, 32, 253-260. https://doi.org/10.1016/j.chb.2013.12.009

Lenhart, A. (2015, August 6). Teens, technology, and friendship. Pew Research Center. Retrieved from http://www.pewinternet.org/2015/08/06/teens-technology-and-friendships/

Marengo, D., Longobardi, C., Fabris, M. A., \& Settanni, M. (2018). Highly-visual social media and internalizing symptoms in adolescence: The mediating role of body image concerns. Computers in Human Behavior, 82, 63-69. https://doi.org/10.1016/j.chb.2018.01.003

Muthén, L. K., \& Muthén, B. O. (2017). Mplus user guide (8th ed.). Los Angeles, CA: Muthén \& Muthén.

Myers, T. A., \& Crowther, J. H. (2009). Social comparison as a predictor of body dissatisfaction: A meta-analytic review. Journal of Abnormal Psychology, 118, 683-698. https://doi.org/10.1037/a0016763

Neemann, J., \& Harter, S. (2012). Self-perception profile for college students: Manual and questionnaires. (Unpublished Manual). University of Denver, Denver, CO.

Nesi, J. (2014). The relationship between adolescent technology use and depressive symptoms: An integrative model of offline and technology-based risk factors. (Unpublished Master's thesis). University of North Carolina at Chapel Hill, Chapel Hill, North Carolina.

Nesi, J., Choukas-Bradley, S., \& Prinstein, M. J. (2018). Transformation of adolescent peer relations in the social media context: Part 2-Application to peer group processes and future directions for research. Clinical Child and Family Psychology Review, 21, 295-319. https://doi.org/10.1007/s10567-018-0262-9

Nesi, J., \& Prinstein, M. J. (2015). Using social media for social comparison and feedback-seeking: Gender and popularity moderate associations with depressive symptoms. Journal of Abnormal Child Psychology, 43, 14271438. https://doi.org/10.1007/s10802-015-0020-0 
Nesi, J., Miller, A. B., \& Prinstein, M. J. (2017). Adolescents' depressive symptoms and subsequent technologybased interpersonal behaviors: A multi-wave study. Journal of Applied Developmental Psychology, 51, 12-19. https://doi.org/10.1016/j.appdev.2017.02.002

Pempek, T. A., Yermolayeva, Y. A., \& Calvert, S. L. (2009). College students' social networking experiences on Facebook. Journal of Applied Developmental Psychology, 30, 227-238. https://doi.org/10.1016/j.appdev.2008.12.010

Perrin, A., \& Anderson, M. (2019, April 10). Share of U.S. adults using social media, including Facebook, is mostly unchanged since 2018. Pew Research Center. Retrieved from https://www.pewresearch.org/facttank/2019/04/10/share-of-u-s-adults-using-social-media-including-facebook-is-mostly-unchanged-since-2018/

Piwek, L., \& Joinson, A. (2016). "What do they snapchat about ?" Patterns of use in time-limited instant messaging service. Computers in Human Behavior, 54, 358-367. https://doi.org/10.1016/j.chb.2015.08.026

Przybylski, A. K., Murayama, K., DeHaan, C. R., \& Gladwell, V. (2013). Motivational, emotional, and behavioral correlates of fear of missing out. Computers in Human Behavior, 29, 1841-1848.

https://doi.org/10.1016/j.chb.2013.02.014

Reer, F., Tang, W. Y., \& Quandt, T. (2019). Psychosocial well-being and social media engagement: The mediating roles of social comparison orientation and fear of missing out. New Media \& Society, 1-20. Advance Online Publication.

Reich, S. M. (2017). Connecting offline social competence to online peer interactions. Psychology of Popular Media Culture, 6, 291-310. https://doi.org/10.1037/ppm0000111

Rousseau, A., Eggermont, S., \& Frison, E. (2017). The reciprocal and indirect relationships between passive Facebook use, comparison on Facebook, and adolescents' body dissatisfaction. Computers in Human Behavior, 73 , 336-344. https://doi.org/10.1016/j.chb.2017.03.056

Shensa, A., Sidani, J. E., Lin, L. Y., Bowman, N. D., \& Primack, B. A. (2016). Social media use and perceived emotional support among US young adults. Journal of Community Health, 41, 541-549.

https://doi.org/10.1007/s10900-015-0128-8

Smith, R. H. (2000). Assimilative and contrastive emotional reactions to upward and downward social comparisons. In J. Suls and L. Wheeler (Eds), Handbook of Social Comparison (pp. 173-200). New York, NY: Kluwer Academic / Plenum Publishers.

Stapleton, P., Luiz, G., \& Chatwin, H. (2017). Generation validation: The role of social comparison in use of Instagram among emerging adults. Cyberpsychology, Behavior, and Social Networking, 20, 142-149.

https://doi.org/10.1089/cyber.2016.0444

Tandoc, E. C., Ferrucci, P., \& Duffy, M. (2015). Facebook use, envy, and depression among college students: Is facebooking depressing? Computers in Human Behavior, 43, 139-146. https://doi.org/10.1016/j.chb.2014.10.053

Underwood, M. K., \& Ehrenreich, S. E. (2017). The power and the pain of adolescents' digital communication: Cyber victimization and the perils of lurking. American Psychologist, 72, 144-158.

https://doi.org/10.1037/a0040429

Valenzuela, S., Park, N., \& Kee, K. F. (2009). Is there social capital in a social network site?: Facebook use and college student's life satisfaction, trust, and participation. Journal of Computer-Mediated Communication, 14, 875901. https://doi.org/10.1111/j.1083-6101.2009.01474.x

Verduyn, P., Lee, D. S., Park, J., Shablack, H., Orvell, A., Bayer, J., ... Kross, E. (2015). Passive facebook usage undermines affective well-being: Experimental and longitudinal evidence. Journal of Experimental Psychology: General, 144, 480-488. https://doi.org/10.1037/xge0000057 
Verduyn, P., Ybarra, O., Résibois, M. Jonides, J., \& Kross, E. (2017). Do social network sites enhance or undermine subjective well-being? A critical review. Social Issues and Policy Review, 11, 274-302.

https://doi.org/10.1111/sipr.12033

Vogel, E. A., \& Rose, J. P. (2016). Self-reflection and interpersonal connection: Making the most of selfpresentation on social media. Trans/ational Issues in Psychological Science, 2, 294-302.

https://doi.org/10.1037/tps0000076

Vogel, E. A., Rose, J. P., Okdie, B. M., Eckles, K., \& Franz, B. (2015). Who compares and despairs? The effect of social comparison orientation on social media use and its outcomes. Personality and Individual Differences, 86 , 249-256. https://doi.org/10.1016/j.paid.2015.06.026

Vogel, E. A., Rose, J. P., Roberts, L. R., \& Eckles, K. (2014). Social comparison, social media, and self-esteem. Psychology of Popular Media Culture, 3, 206-222. https://doi.org/10.1037/ppm0000047

Whillans, A. V., \& Chen, F. S. (2018). Facebook undermines the social belonging of first year students. Personality and Individual Differences, 133, 13-16. https://doi.org/10.1016/j.paid.2017.03.043

Whillans, A. V., Christie, C. D., Cheung, S., Jordan, A. H., \& Chen, F. S. (2017). From misperception to social connection: Correlates and consequences of overestimating others' social connectedness. Personality and Social Psychology Bulletin, 43, 1696-1711. https://doi.org/10.1177/0146167217727496

Wilson, R. E., Gosling, S. D., \& Graham, L. T. (2012). A review of Facebook research in the social sciences. Perspectives on Psychological Science, 7, 203-220. https://doi.org/10.1177/1745691612442904

\section{Correspondence to:}

Kaitlyn Burnell

School of Behavioral and Brain Sciences, GR41

The University of Texas at Dallas

800 W Campbell Road

Richardson, TX 75080

Email: Kaitlyn.Burnell(at)utdallas.edu

Editorial record: First submission received on May 10, 2018. Revisions received on December 19, 2018, and May 30 , 2019. Accepted for publication on June 26, 2019.

Editor in charge: Michel Walrave 


\section{About Authors}

Kaitlyn Burnell, M.S., is a doctoral student in the Psychological Sciences program at the University of Texas at Dallas. She is interested in the effects of social media use upon well-being, especially in the context of classic social and developmental psychological theories.

Madeleine J. George, Ph.D., is a post-doctoral research associate at Purdue University. Her research focuses on how adolescents' and young adults' usage of digital technologies may influence their well-being, especially with regards to the development of relationships and feelings of social support.

Justin W. Vollet, Ph.D., is a post-doctoral research associate at the University of Texas at Dallas. Broadly, his research interests center on understanding how psychological and social development during childhood and adolescence take shape through ongoing social interactions with others (i.e., peers, teachers, and parents), with specific focus on how emerging digital media and communications technologies may affect these social processes.

Samuel E. Ehrenreich, Ph.D., is an Assistant Professor in the Human Development and Family Studies program at the University of Nevada, Reno. His research interests focus on how digital communication shapes adolescents' development and relationships.

Marion K. Underwood, Ph.D., is a Professor of Psychology and Dean of Health and Human Services at Purdue University. Her research focuses on the development and outcomes of social aggression, and how digital communication affects peer relationships and adjustment. 